\title{
Combined analysis of gene expression, miRNA expression and DNA methylation profiles of osteosarcoma
}

\author{
WENPENG ZHANG ${ }^{1,2}$, SHILIANG HAN ${ }^{2}$ and KANG SUN ${ }^{1}$ \\ ${ }^{1}$ Department of Joint Surgery, The Affiliated Hospital of Qingdao University, Qingdao, Shandong 266003; \\ ${ }^{2}$ Department of Orthopaedics, Zibo Central Hospital, Zibo, Shandong 255036, P.R. China
}

Received July 28, 2016; Accepted December 6, 2016

DOI: 10.3892/or.2016.5324

\begin{abstract}
Osteosarcoma (OS) is a common primary malignancy in children and adolescents with relative high survival rate after chemotherapy. While the toxicity of chemotherapy and personalized different response to chemotherapy makes it difficult for the selection of therapeutics and improvement of diagnosis. In this study, we conducted a combined analysis of three types of microarray datasets (gene expression, microRNA (miRNA) expression and DNA methylation) from the Gene Expression Omnibus (GEO). The differential expression genes (DEGs) and miRNAs (DEMI) were screened out via the limma package and differential methylation sites (DMS) were identified by the IMA package. Enriched functions of DEGs and genes contained DMS (DEMs) were obtained through the Database for Annotation, Visualization and Integrated Discovery (DAVID). Besides, miRNA-gene regulation network was obtained based on the pairs of involved DEMIs and overlapping genes between DEMs and DEGs and visualized through Cytoscape software. A total of 583 DEGs and 1051 DMS (corresponding to 827 DEMs) were identified and 56 overlaps were obtained. As expected, most of the expression and methylation profiles of the overlaps exhibited significant negative correlation. The DEGs were mainly enriched in the biological processes related to inflammatory/immune response, cell proliferation, while DEMs were involved in the regulation of gene expression, tissue/organ development. Based on the correlation and network analysis, some novel targets were identified for OS and many known biomarkers were proved in this study, which would be helpful in its early diagnosis and treatment.
\end{abstract}

\section{Introduction}

Osteosarcoma (OS) is a common primary malignancy of bone that occurs mostly in children or adolescents with more than

Correspondence to: Professor Kang Sun,Department of Joint Surgery, The Affiliated Hospital of Qingdao University, 16 Jiangsu Road, Qingdao, Shandong 266003, P.R. China

E-mail: sunkangqd@126.com

Key words: ostesarcoma, DNA methylation, GEO, biomarker
26,400 new cases every year $(1,2)$. Chemotherapy and surgery, or their combination, is the prevalent treatment method and has significantly improved the prognosis of OS $(3,4)$. For example, the five-year survival rate of OS without distal metastasis after chemotherapy could reach 70\% (5). While, poor prognosis was frequently observed in the metastatic patients. Moreover, the toxicity of some chemotherapeutic drugs cannot be efficiently prevented and their efficacy could be largely different in similar patients $(6,7)$. Therefore, early diagnosis and personalized therapy would be the alternative methods for the improvement of prognosis of primary as well as the metastatic OS.

In the past decades, many biomarkers have been identified to be associated with the progression of OS and one of the most well known targets is mammalian target of rapamycin (mTOR), a serine/threonine protein kinase, which could contribute to the development of many types of cancers (8). mTOR was regulated by various molecules in OS, downregulation of RSK2 was associated with the inactivating of mTOR signaling pathways and could influence the progression of OS (9); the variation of microRNA-99a could induce the differential expression of mTOR in OS and could influence its development (10). Besides, the mutations of some genes were also shown to affect the susceptibility of OS, such as the polymorphisms of murine double minute 2 (MDM2) (rs1690916 and rs2279744) were associated with the risk of OS (11); the mutation of P15 gene was significantly associated with the initiation of OS (12). In addition, some inhibitors have been developed against these biomarkers. Icariside II is a natural mTOR inhibitor which could destroy the energy balance through inhibiting mTORC1-4E-BP1 axis to inhibit the progression of sarcoma (13). While, further studies are still needed for the understanding of the mechanisms of OS and the improvement of its prognosis.

In this study, the DNA methylation profile was also included besides the gene and microRNA (miRNA) expression profiles, which play important roles in the regulation of gene expression. DNA hypermethylation in promoter is a key characteristic in cancer and it was considered to be able to silence the tumor suppressor genes to induce the development of cancer (14-16). Some DNA methylation was also observed in OS and found to be associated with its progression, the methylation level of RECK was gradually increased with the development of OS, while the opposite trend of its expres- 
sion profile was observed (17). In the study of Sonaglio et al, the differential ESR1 and p14ARF gene methylation profiles were also identified as prognostic indicators in OS (18). The combined analysis of gene, miRNA expression and DNA methylation profiles would be helpful for the identification of novel biomarkers for OS and its early diagnosis and treatment.

\section{Material and methods}

Microarray datasets. The microarray datasets in this study were downloaded from the Gene Expression Omnibus (GEO, http://www.ncbi.nlm.nih.gov/geo/). The gene expression (GSE36001) and DNA methylation profiles (GSE36002) were deposited by Kresse et al and composed with the same samples: 19 OS cell lines (case group) and 6 normal samples (control group, 2 osteoblast and 4 bone samples) (19). While, the miRNA expression profiles (GSE28423) were provided by Namløs et al, compared with the gene expression and DNA methylation profiles, only 2 osteoblast were absent and the other samples were exactly the same, i.e. 19 OS cell lines (case group) and 4 normal bone samples (control group) (20). The samples for the three types of profiles were comparable and could be used for the subsequent analysis.

Microarray analysis. R Bioconductor packages were used for the preprocessing of the three types of microarray datasets. For gene and miRNA expression profiles, raw datasets were normalized based on the preprocess Core package and the differential expression genes (DEGs) and miRNAs (DEMIs) were screened out via the limma package through the thresholds of fold change $>2$ or $<0.5$ and adjusted P-value of $<0.05$. IMA package was adopted for the preprocessing of raw DNA methylation profile and the identification of differential methylation sites (DMS) and in this study, the methylation sites satisfied the criteria of $\log 2$ (fold change) $>0.5$ or $<-0.5$ and adjusted $\mathrm{P}$-value $<0.05$ were screened out.

Functional enrichment analysis. Based on the Database for Annotation, Visualization and Integrated Discovery (DAVID, http://david.abcc.ncifcrf.gov), the Gene Ontology (GO) terms and Kyoto Encyclopedia of Genes and Genomes (KEGG) pathways of DEGs and genes contained DMS (DEMs) were obtained. The thresholds used here is P-value $<0.05$.

miRNA-gene regulation network. Besides the promoter methylation, miRNA is another important regulator for gene expression. In this study, the target genes of DEMIs were identified through the TargetScan, a database of the miRNAgene pairs obtained by various methods, such as biological experiments and bioinformatics prediction. Furthermore, we selected only the pairs that contained the DEMIs and overlapping genes of DEGs and DEMs. The Cytoscape, a network visualization and analysis software, was used for the construction of miRNA-gene regulation network.

\section{Results}

DEGs, DEMIs and DMS. Compared with the control group, a total of 583 genes were found to be differentially expressed in the case group, which contained 417 downregulated and
Table I. Top 10 significantly differential expression genes and methylated sites.

\begin{tabular}{lrcccc}
\hline DEG & Log2FC & Adj.P.Value & DMS & Log2FC & Adj.P.Value \\
\hline CMKLR1 & -1.03 & $1.16 \times 10^{-8}$ & $\operatorname{cg} 03874199$ & 0.90 & $1.42 \times 10^{-22}$ \\
PTGS1 & -1.67 & $3.62 \times 10^{-8}$ & $\operatorname{cg} 18765542$ & 0.87 & $2.07 \times 10^{-20}$ \\
RPS28 & 1.15 & $5.31 \times 10^{-8}$ & $\operatorname{cg} 23130254$ & 0.86 & $9.85 \times 10^{-19}$ \\
HOXB7 & 2.09 & $9.50 \times 10^{-8}$ & $\operatorname{cg} 06274159$ & 0.84 & $5.82 \times 10^{-18}$ \\
UQCRHL & 1.91 & $9.59 \times 10^{-8}$ & $\operatorname{cg} 09522147$ & 0.63 & $3.07 \times 10^{-16}$ \\
RHPN2 & 2.15 & $4.70 \times 10^{-7}$ & $\operatorname{cg} 01615704$ & 0.83 & $3.89 \times 10^{-16}$ \\
RPS27 & 1.33 & $2.73 \times 10^{-6}$ & $\operatorname{cg} 00208967$ & 0.73 & $4.01 \times 10^{-16}$ \\
SRGN & -5.17 & $2.73 \times 10^{-8}$ & $\operatorname{cg} 08668790$ & 0.87 & $1.73 \times 10^{-15}$ \\
FOXF2 & 2.32 & $3.64 \times 10^{-8}$ & $\operatorname{cg} 09009380$ & 0.67 & $1.78 \times 10^{-15}$ \\
RPL23 & 1.70 & $4.87 \times 10^{-8}$ & $\operatorname{cg} 22396755$ & 0.82 & $1.99 \times 10^{-15}$ \\
\hline
\end{tabular}

Table II. Top 10 significantly differential expression miRNAs.

\begin{tabular}{lcc}
\hline DEMI & Log2FC & Adj.P.Value \\
\hline hsa-miR-144 & -6.45 & $5.94 \times 10^{-16}$ \\
hsa-miR-638 & -3.66 & $1.43 \times 10^{-12}$ \\
hsa-miR-1225-5p & -4.84 & $1.43 \times 10^{-12}$ \\
hsa-miR-451 & -14.77 & $1.30 \times 10^{-11}$ \\
hcmv-miR-UL70-3p & -4.84 & $8.48 \times 10^{-11}$ \\
hsa-miR-486-5p & -5.36 & $1.20 \times 10^{-10}$ \\
hsv1-miR-LAT & -4.54 & $3.19 \times 10^{-10}$ \\
hsa-miR-188-5p & -3.94 & $3.19 \times 10^{-10}$ \\
hsa-miR-671-5p & -4.80 & $5.21 \times 10^{-10}$ \\
hsa-miR-134 & -4.37 & $6.15 \times 10^{-10}$ \\
\hline
\end{tabular}

166 upregulated genes. Besides, we identified 1051 DMS in the case group which corresponding to 827 unique genes and strikingly, 1018 DMS, 96.9\% of all the DMS, were found to be hypermethylation. In total, 231 DEMIs were identified in case group and 117 were found to be downregulated. The top 10 significantly differentially expressed genes and methylation sites are shown in Table I and in Table II the top 10 significantly differentially expressed miRNAs. The supervised hierarchical cluster of DEGs and DMS based on gplots package are shown in Fig. 1. The supervised cluster of DEMIs are shown in Fig. 2. As shown in Figs. 1 and 2, the OS and control samples can be separated into their types, which indicated the reliability of the DEGs, DEMIs and DMS for the following analysis.

Correlation of gene expression and DNA methylation profiles. A total of 56 overlaps were identified between DEGs and DEMs. The supervised cluster of the overlapping genes and their corresponding DMS are shown in Fig. 3. As expected, significant negative correlation with the minimum of -0.924 was observed between most of the methylation profiles of DMS and their corresponding expression profile of the overlaps. Fig. 4 illustrates the distribution of the correlation coefficients. 
A

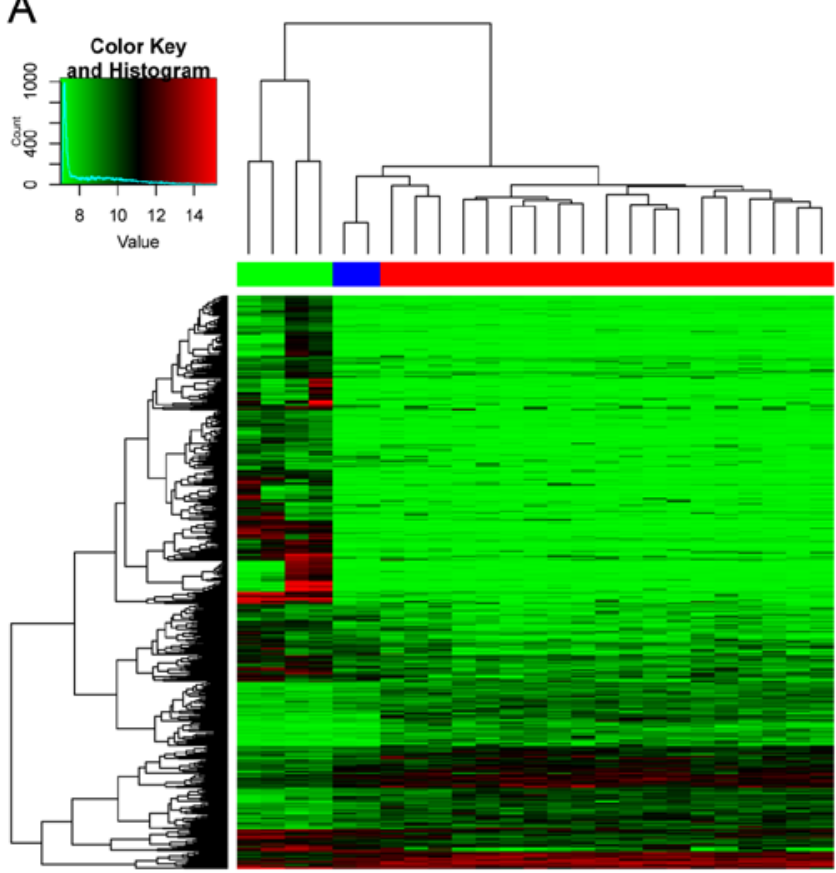

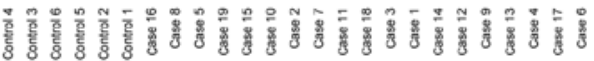
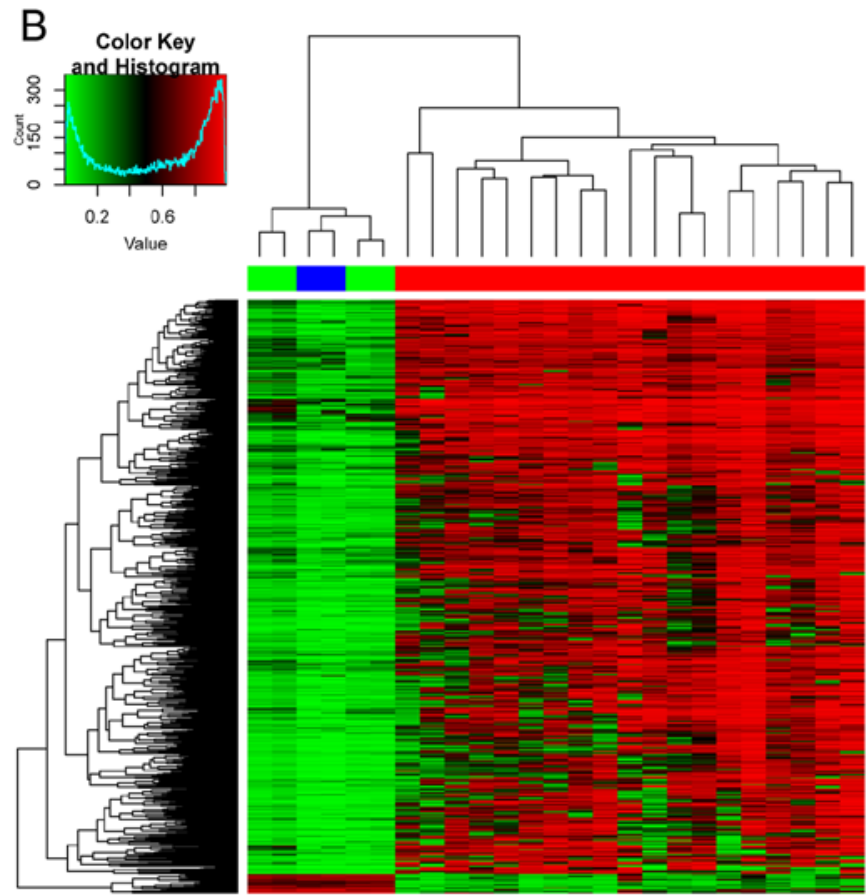

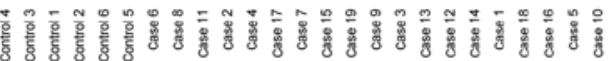

Figure 1. Supervised cluster of DEGs (A) and DMS (B).
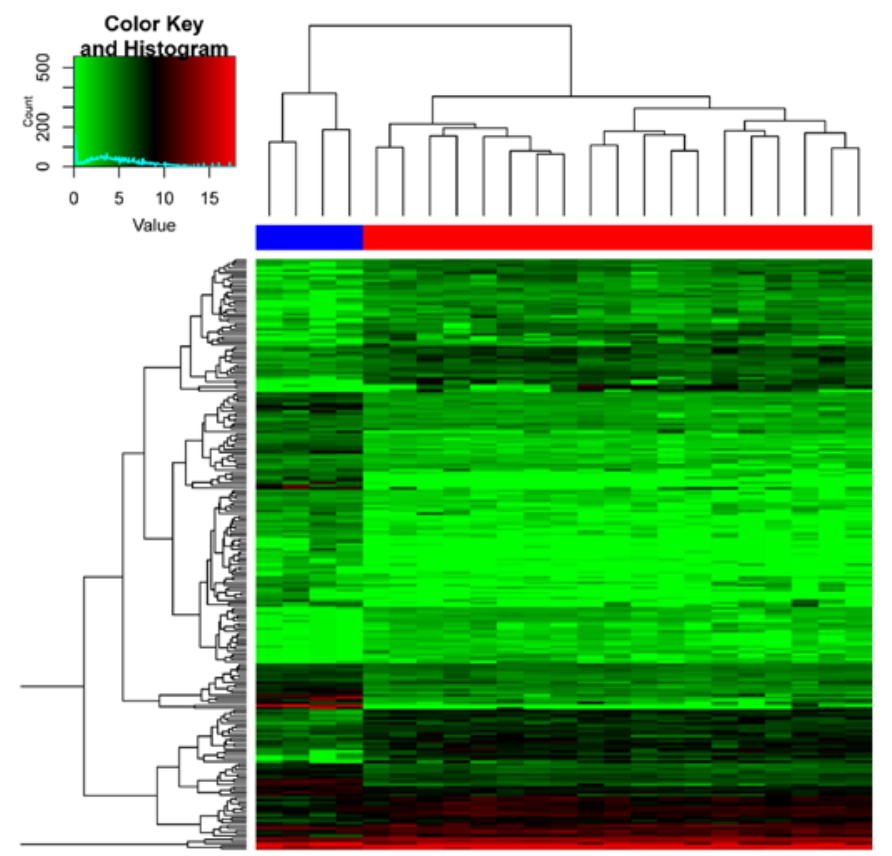

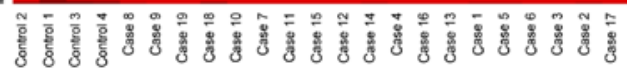

Figure 2. Supervised cluster of DEMIs.

Enriched functions. A total of 180 and 198 GO terms were found to be enriched in the DEGs and DEMs, respectively and the top 20 significantly enriched GO terms of DEGs and DEMs are shown in Fig. 5. The cellular component of plasma/cytoplasm were found to be enriched in both DEGs and DEMs. While the biological processes related to inflammatory/immune response, cell proliferation, and so on, were
Table III. KEGG pathways enriched in DEGs.

\begin{tabular}{lrl}
\hline Pathway name & Count & P-value \\
\hline Asthma & 11 & $3.40 \times 10^{-7}$ \\
Intestinal immune network for IgA production & 10 & $3.30 \times 10^{-4}$ \\
Viral myocarditis & 12 & $3.60 \times 10^{-4}$ \\
Glycine, serine and threonine metabolism & 8 & $4.20 \times 10^{-4}$ \\
Hematopoietic cell lineage & 13 & $5.20 \times 10^{-4}$ \\
Systemic lupus erythematosus & 14 & $5.60 \times 10^{-4}$ \\
Leukocyte transendothelial migration & 15 & $9.80 \times 10^{-4}$ \\
Cell adhesion molecules (CAMs) & 16 & $1.00 \times 10^{-3}$ \\
Allograft rejection & 8 & $1.10 \times 10^{-3}$ \\
Graft-versus-host disease & 8 & $1.80 \times 10^{-3}$ \\
Type I diabetes mellitus & 8 & $2.80 \times 10^{-3}$ \\
Natural killer cell mediated cytotoxicity & 15 & $3.10 \times 10^{-3}$ \\
Complement and coagulation cascades & 10 & $4.10 \times 10^{-3}$ \\
Autoimmune thyroid disease & 8 & $8.40 \times 10^{-3}$ \\
Nitrogen metabolism & 5 & $2.00 \times 10^{-2}$ \\
One carbon pool by folate & 4 & $3.50 \times 10^{-2}$ \\
Antigen processing and presentation & 9 & $3.70 \times 10^{-2}$ \\
Tight junction & 12 & $4.50 \times 10^{-2}$ \\
Pathogenic Escherichia coli infection & 7 & $4.60 \times 10^{-2}$
\end{tabular}

enriched in DEGs, and DEMs were mainly associated with the biological process of regulation of gene expression, tissue and embryonic development. Besides, 19 and 6 KEGG pathways were also enriched in DEGs and DEMs, respectively (Tables III 

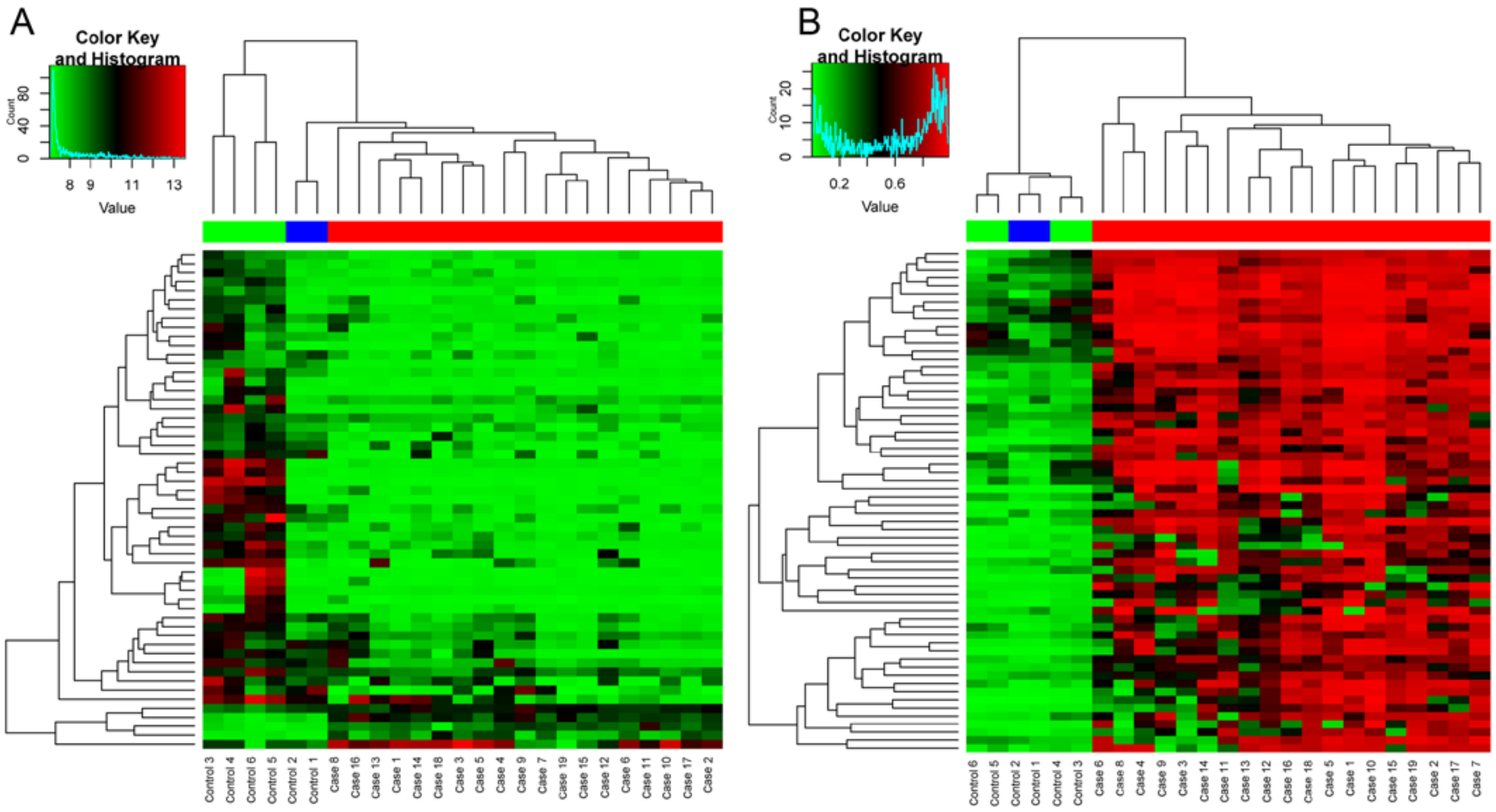

Figure 3. Supervised cluster of expression (A) and methylation (B) profiles of the overlapping genes of DEGs and DEMs.

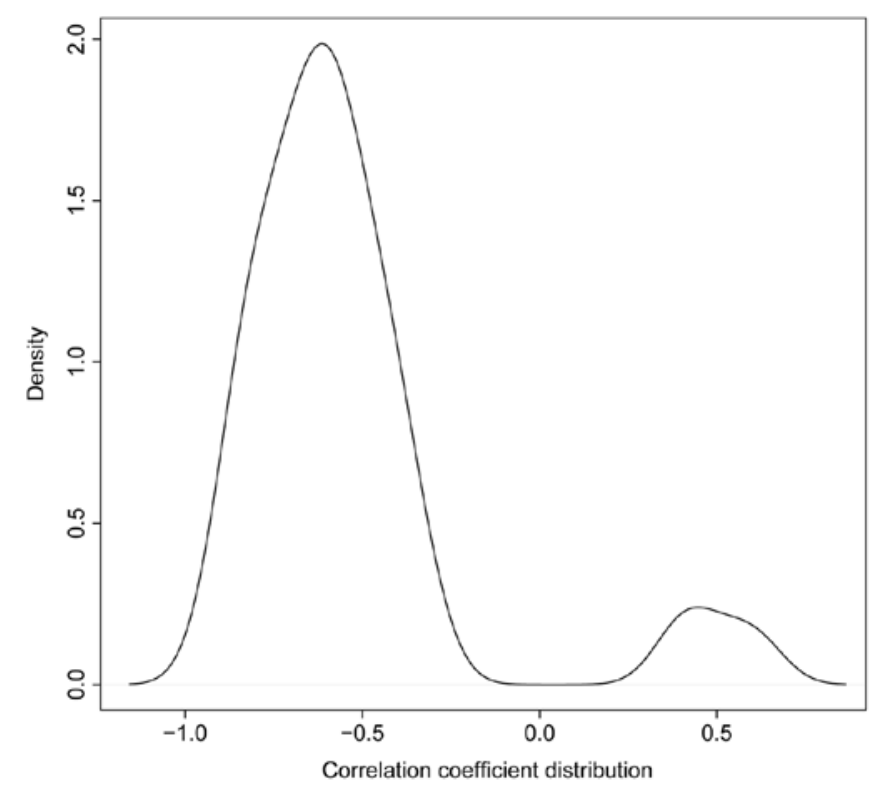

Figure 4. Correlation coefficient distribution of expression and methylation profiles of the overlapping genes of DEGs and DEMs.

and IV). Similar with the GO terms, KEGG pathways of DEGs were mainly involved in inflammatory and immune processes, while the enriched pathways of DEMs were related to cancer development and cytokine signaling pathways.

miRNA-gene regulation network. Through the TargetScan, we screened out 8596 miRNA-gene pairs that contained DEMIs, and 58 pairs of which were found to be involved the overlapping genes of DEGs and DEMs. The miRNA-gene regulation
Table IV. KEGG pathways enriched in DEMs.

\begin{tabular}{lcc}
\hline Pathway name & Count & P-value \\
\hline Neuroactive ligand-receptor interaction & 27 & $2.86 \times 10^{-4}$ \\
Pathways in cancer & 29 & $2.62 \times 10^{-3}$ \\
Small cell lung cancer & 10 & $2.13 \times 10^{-2}$ \\
Cytokine-cytokine receptor interaction & 21 & $3.18 \times 10^{-2}$ \\
Amyotrophic lateral sclerosis (ALS) & 7 & $4.41 \times 10^{-2}$ \\
Adipocytokine signaling pathway & 8 & $4.50 \times 10^{-2}$ \\
\hline
\end{tabular}

network composed of the 58 pairs shown in Fig. 6. A total of 34 miRNAs and 24 genes were contained in the network and hsa-miR-128 regulated 5 of the 24 genes. Besides, TIMP4 (TIMP metallopeptidase inhibitor 4), found to be associated with the progression of breast cancer by many studies, was regulated by 7 miRNAs in the miRNA-gene network, that might indicate its important role in OS.

\section{Discussion}

OS is one of the most common malignancies in children and adolescents with a relative high survival rate after chemotherapy or surgery in primary tumors while not in the metastatic ones. Identification of novel biomarkers would be helpful in its early diagnosis and improvement of prognosis. In this study, three types of microarray datasets: gene expression profile, miRNA expression profile and DNA methylation profile were downloaded for the GEO and analyzed for their relationship with the progression of OS. This would benefit the 


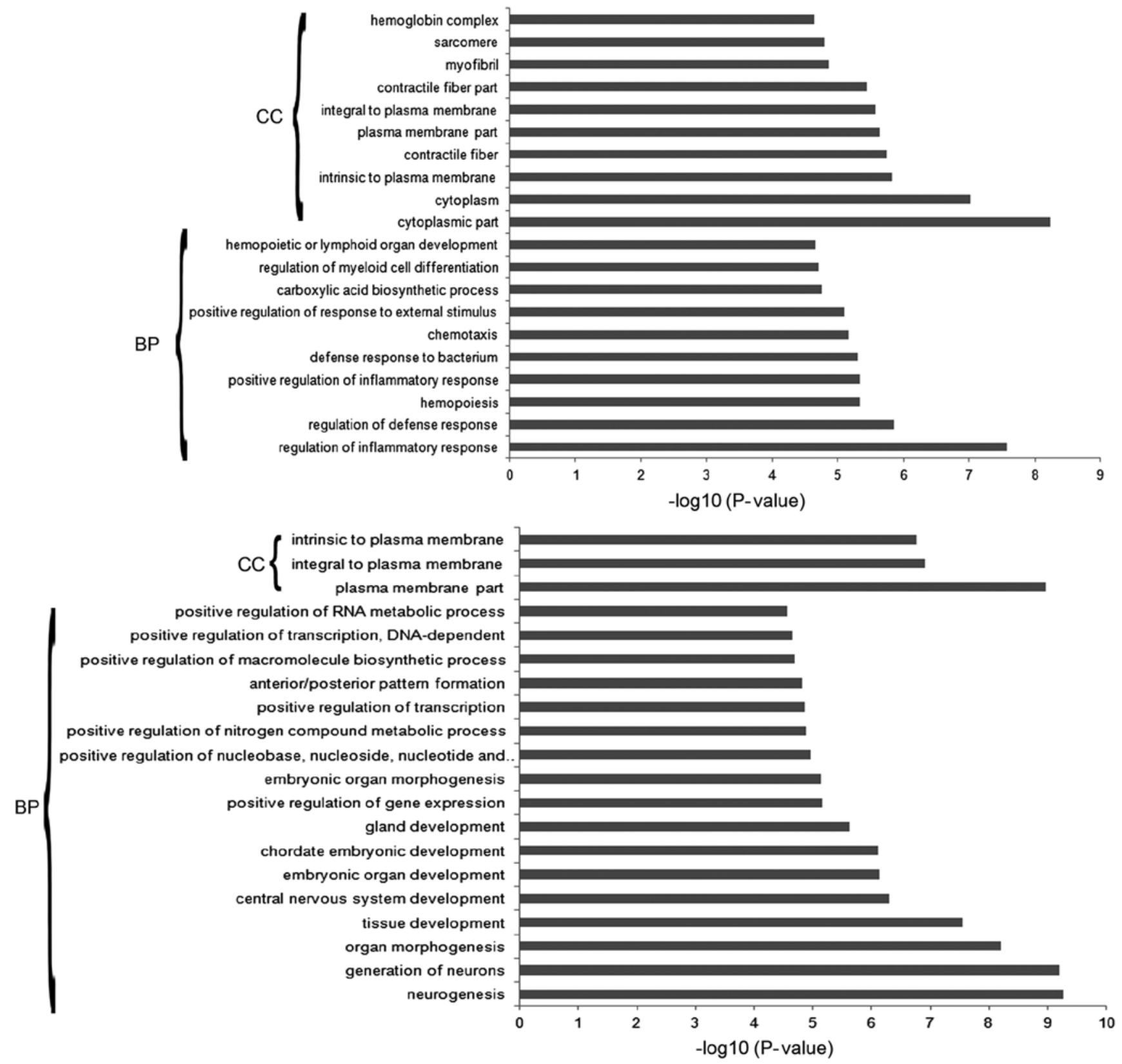

Figure 5. The top 20 enriched GO terms of DEGs and DEMs. CC, cellular component; BP, biological process.

understanding of the mechanisms of OS and the development of novel therapeutics.

Herein, $71.5 \%$ and $3.1 \%$ DEGs and DMS were found to be downregulated in the OS group compared with the control ones respectively. The expression profiles and the corresponding methylation profiles of the overlapping genes between DEGs and DEMs exhibited striking negative correlation as shown in Fig. 4, which might indicate their antitumor role. In particular, LXN (latexin), a gene encoding the only known protein inhibitor of zinc-dependent metallocarboxypetidases, were found to be downregulated in many types of tumors, as well as in OS in our study and it exhibited significantly negative correlation between expression and methylation profile (cor -0.92, P-value $\left.4.25 \times 10^{-11}\right)$. In hepatocellular carcinoma $(21,22)$, gastric carcinomas (23), pancreatic cancer (24) and thyroid cancer (25), LXN was considered to exhibits tumor suppressor while not in
OS, so it might be a novel target for the diagnosis or treatment of OS. Besides, CMKLR1 (chemokine-like receptor 1), exhibited downregulation and hypermethylation simultaneously in OS group, and was identified to affect the treatment of chemotherapy in non-small cell lung cancer (26) and the endothelial angiogenesis (27), which might also influence the development of OS. Some known biomarkers were also identified in this study, such as RAC2 (28) and CD248 (29), which indicated the reliability of our study.

The DEGs and DEMs were found to be enriched in entirely different aspects of the biological process of ontology, e.g. the DEGs were mainly involved in inflammatory/immune response, and cell proliferation related processes, while DEMs were associated with the processes of regulation of gene expression, tissue and embryonic development. However, all of the terms are closely associated with 


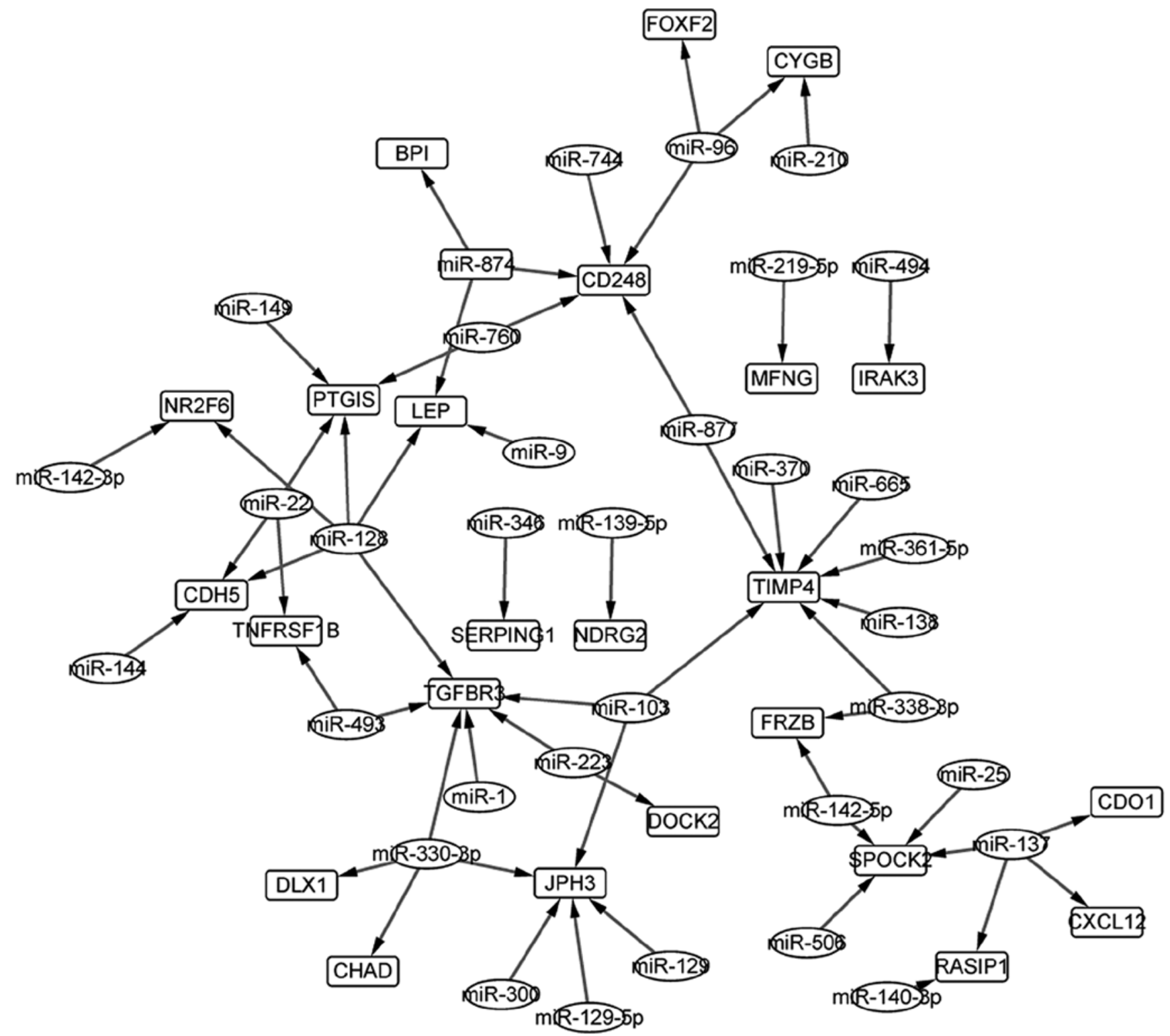

Figure 6. miRNA-gene regulation network. Ovals represent miRNAs and rectangles are genes.

the development of many types of cancers. Moreover, DNA methylation could lead to altered gene expression and regulation and thus affect the progression of cancers (30-32). Our study also supported the findings and expected to be helpful in the understanding of the mechanisms of OS.

In this study, potential miRNA regulators of overlapping genes between DEGs and DEMs were screened out through the TargetScan online software and the miRNA expression profile dataset of OS. Finally, the miRNA-gene regulation network was obtained in which TIMP4 (TIMP metallopeptidase inhibitor 4) was directly regulated by 7 miRNAs. TIMP4 was downregulated in the OS group and exhibited significantly negative correlation between gene expression and methylation profiles (cor -0.66 , P-value $3.70 \times 10^{-4}$ ). It was shown to play important roles in the extracellular matrix and the progression of cancers, such as cervical cancer (33), however, no study has associated it with OS, which might indicate it as a novel target. Besides, miRNA-128, which has been shown to influence the risk of OS by many studies (34-36), regulated more genes than the other miRNAs in the miRNA-gene regulation network demonstrating the conformance of our study with the previous ones, besides the novel findings.

In conclusion, the combined analysis of three types of microarray datasets identified some novel biomarkers for OS and many known ones were also confirmed in this study. Our results are helpful for the diagnosis and treatment of OS and thus improvement of prognosis.

\section{References}

1. Bishop MW and Janeway KA: Emerging concepts for PI3K/mTOR inhibition as a potential treatment for osteosarcoma. F1000Res 5: pii: F1000, 2016. doi: 10.12688/f1000research.8228.1.

2. Picci P: Osteosarcoma (osteogenic sarcoma). Orphanet J Rare Dis 2: 6, 2007.

3. Whelan J, Seddon B and Perisoglou M: Management of osteosarcoma. Curr Treat Options Oncol 7: 444-455, 2006.

4. Longhi A, Errani C, De Paolis M, Mercuri M and Bacci G: Primary bone osteosarcoma in the pediatric age: State of the art. Cancer Treat Rev 32: 423-436, 2006. 
5. Wan J, Zhang X, Liu T and Zhang X: Strategies and developments of immunotherapies in osteosarcoma. Oncol Lett 11: 511-520, 2016.

6. Vos HI, Coenen MJ, Guchelaar HJ and Te Loo DM: The role of pharmacogenetics in the treatment of osteosarcoma. Drug Discov Today 21: 1775-1786, 2016.

7. Serra M and Hattinger CM: The pharmacogenomics of osteosarcoma. Pharmacogenomics J: May 31, 2016 (Epub ahead of print). doi: $10.1038 /$ tpj.2016.45

8. Hu K, Dai HB and Qiu ZL: mTOR signaling in osteosarcoma: Oncogenesis and therapeutic aspects (Review). Oncol Rep 36 : 1219-1225, 2016.

9. Qiu Q, Jiang J, Lin L, Cheng S, Xin D, Jiang W, Shen J and Hu Z: Downregulation of RSK2 influences the biological activities of human osteosarcoma cells through inactivating AKT/mTOR signaling pathways. Int J Oncol 48: 2508-2520, 2016.

10. Zhao J, Chen F, Zhou Q, Pan W, Wang X, Xu J, Ni L and Yang H: Aberrant expression of microRNA-99a and its target gene mTOR associated with malignant progression and poor prognosis in patients with osteosarcoma. Onco Targets Ther 9: 1589-1597, 2016.

11. Bilbao-Aldaiturriaga N, Askaiturrieta Z, Granado-Tajada I, Goričar K, Dolžan V, Garcia-Miguel P, Garcia de Andoin N, Martin-Guerrero I and Garcia-Orad A; For The Slovenian Osteosarcoma Study Group: A systematic review and meta-analysis of MDM2 polymorphisms in osteosarcoma susceptibility. Pediatr Res 80: 472-479, 2016.

12. Yu C and Wang W: Relationship between P15 gene mutation and formation and metastasis of malignant osteosarcoma. Med Sci Monit 22: 656-661, 2016.

13. Zhang C, Yang L, Geng YD, An FL, Xia YZ, Guo C, Luo JG, Zhang LY, Guo QL and Kong LY: Icariside II, a natural mTOR inhibitor, disrupts aberrant energy homeostasis via suppressing mTORC1-4E-BP1 axis in sarcoma cells. Oncotarget 7: 2781927837, 2016.

14. Kalmár A, Péterfia B, Hollósi P, Galamb O, Spisák S, Wichmann B, Bodor A, Tóth K, Patai ÁV, Valcz G, et al: DNA hypermethylation and decreased mRNA expression of MAL, PRIMA1, PTGDR and SFRP1 in colorectal adenoma and cancer. BMC Cancer 15: 736, 2015

15. Qiu X, Hu B, Huang Y, Deng Y, Wang $X$ and Zheng $F$ : Hypermethylation of ACP1, BMP4, and TSPYL5 in hepatocellular carcinoma and their potential clinical significance. Dig Dis Sci 61: 149-157, 2016.

16. Choudhury JH and Ghosh SK: Promoter hypermethylation profiling identifies subtypes of head and neck cancer with distinct viral, environmental, genetic and survival characteristics. PLoS One 10: e0129808, 2015.

17. Wang L, Ge J, Ma T, Zheng Y, Lv S, Li Y and Liu S: Promoter hypermethylation of the cysteine protease RECK may cause metastasis of osteosarcoma. Tumour Biol 36: 9511-9516, 2015

18. Sonaglio V, de Carvalho AC, Toledo SR, Salinas-Souza C, Carvalho AL, Petrilli AS, de Camargo B and Vettore AL: Aberrant DNA methylation of ESR1 and p14ARF genes could be useful as prognostic indicators in osteosarcoma. Onco Targets Ther 6: 713-723, 2013.

19. Kresse SH, Rydbeck H, Skårn M, Namløs HM, BarraganPolania AH, Cleton-Jansen AM, Serra M, Liestøl K, Hogendoorn PC, Hovig E, et al: Integrative analysis reveals relationships of genetic and epigenetic alterations in osteosarcoma. PLoS One 7: e48262, 2012
20. Namløs HM, Meza-Zepeda LA, Barøy T, Østensen IH, Kresse SH, Kuijjer ML, Serra M, Bürger H, Cleton-Jansen AM and Myklebost O: Modulation of the osteosarcoma expression phenotype by microRNAs. PLoS One 7: e48086, 2012.

21. Ni QF, Tian Y, Kong LL, Lu YT, Ding WZ and Kong LB: Latexin exhibits tumor suppressor potential in hepatocellular carcinoma. Oncol Rep 31: 1364-1372, 2014.

22. Muthusamy V, Premi S, Soper C, Platt J and Bosenberg M: The hematopoietic stem cell regulatory gene latexin has tumorsuppressive properties in malignant melanoma. J Invest Dermatol 133: 1827-1833, 2013.

23. Li Y, Basang Z, Ding H, Lu Z, Ning T, Wei H, Cai H and Ke Y: Latexin expression is downregulated in human gastric carcinomas and exhibits tumor suppressor potential. BMC Cancer 11: 121, 2011.

24. Xue ZX, Zheng JH, Zheng ZQ, Cai JL, Ye XH, Wang C, Sun WJ, Zhou X, Lu MD, Li PH, et al: Latexin inhibits the proliferation of $\mathrm{CD}_{133^{+}}$miapaca-2 pancreatic cancer stem-like cells. World J Surg Oncol 12: 404, 2014

25. Abd Elmageed ZY, Moroz K and Kandil E: Clinical significance of CD146 and latexin during different stages of thyroid cancer. Mol Cell Biochem 381: 95-103, 2013.

26. Wu X, Ye Y, Rosell R, Amos CI, Stewart DJ, Hildebrandt MA, Roth JA, Minna JD, Gu J, Lin J, et al: Genome-wide association study of survival in non-small cell lung cancer patients receiving platinum-based chemotherapy. J Natl Cancer Inst 103: 817-825, 2011.

27. Kaur J, Adya R, Tan BK, Chen J and Randeva HS: Identification of chemerin receptor (ChemR23) in human endothelial cells: Chemerin-induced endothelial angiogenesis. Biochem Biophys Res Commun 391: 1762-1768, 2010.

28. Han JA, Kim JY and Kim JI: Analysis of gene expression in cyclooxygenase-2-overexpressed human osteosarcoma cell lines. Genomics Inform 12: 247-253, 2014.

29. Sun DX, Liao GJ, Liu KG and Jian H: Endosialin-expressing bone sarcoma stem-like cells are highly tumor-initiating and invasive. Mol Med Rep 12: 5665-5670, 2015.

30. Song L and Li Y: The role of stem cell DNA methylation in colorectal carcinogenesis. Stem Cell Rev 12: 573-583, 2016.

31. Xu P, Hu G, Luo C and Liang Z: DNA methyltransferase inhibitors: An updated patent review (2012-2015). Expert Opin Ther Pat 26: 1017-1030, 2016.

32. Xie P, Zang LQ, Li XK and Shu Q: An epigenetic view of developmental diseases: New targets, new therapies. World J Pediatr 12: 291-297, 2016.

33. Lizarraga F, Ceballos-Cancino G, Espinosa M, VazquezSantillan K, Maldonado V and Melendez-Zajgla J: Tissue inhibitor of metalloproteinase-4 triggers apoptosis in cervical cancer cells. PLoS One 10: e0135929, 2015

34. Liu X, Liang Z, Gao K, Li H, Zhao G, Wang S and Fang J: MicroRNA-128 inhibits EMT of human osteosarcoma cells by directly targeting integrin alpha2. Tumour Biol 37: 7951-7957, 2016.

35. Tian Z, Guo B, Yu M, Wang C, Zhang H, Liang Q, Jiang K and Cao L: Upregulation of micro-ribonucleic acid-128 cooperating with downregulation of PTEN confers metastatic potential and unfavorable prognosis in patients with primary osteosarcoma. Onco Targets Ther 7: 1601-1608, 2014

36. Shen L, Chen XD and Zhang YH: MicroRNA-128 promotes proliferation in osteosarcoma cells by downregulating PTEN. Tumour Biol 35: 2069-2074, 2014. 